\title{
What causes a prolactinoma to be aggressive or to become a pituitary carcinoma?
}

\author{
Jana Phillips, ${ }^{1}$ Honey E. East, ${ }^{1}$ Sarah E. French, ${ }^{1}$ Eugen Melcescu, ${ }^{1}$ \\ Robert D. Hamilton, ${ }^{2}$ William C. Nicholas, ${ }^{1}$ Jonathan F. Fratkin, ${ }^{3}$ \\ Andrew D. Parent, ${ }^{4}$ Gustavo Luzardo, ${ }^{4}$ Christian A. Koch ${ }^{1,5}$
}

${ }^{1}$ Division of Endocrinology, Department of Medicine, ${ }^{2}$ Division of Oncology, Department of Medicine, ${ }^{3}$ Department of Pathology, ${ }^{4}$ Department of Neurosurgery, University of Mississippi Medical Center, ${ }^{5} G V$ (Sonny) Montgomery VA Medical Center, Woodrow Wilson, Jackson, Mississippi, USA

\begin{abstract}
INTRODUCTION: Malignant prolactinoma is an exceedingly rare endocrine tumor and cannot be diagnosed on histological grounds alone. Similarly to other neuroendocrine tumors such as pheochromocytoma, the mitoses index, Ki-67, p53, and others are utilized in helping understand whether a tumor is benign or malignant or to better predict tumor behavior. We here present the unusual case of an unfortunate young man with an aggressive prolactinoma, the complications of which led to his premature death. CASE REPORT: A 25-year-old white man developed severe headaches, low energy, and decreased libido. A brain magnetic resonance imaging (MRI) showed a $4 \times 3 \times 2 \mathrm{~cm}$ pituitary tumor invading the left cavernous sinus. Laboratory findings revealed elevated prolactin $(470 \mathrm{ng} / \mathrm{mL})$ and adrenocorticotropic hormone (ACTH, $82 \mathrm{pg} / \mathrm{ml}$ ) and decreased total testosterone $(176 \mathrm{ng} / \mathrm{dl})$. Visual fields showed superior quadrantanopia in the left eye. Transsphenoidal pituitary resection was undertaken. Pathology revealed a prolactinoma with atypical cells, diffuse p53 nuclear labeling, and a Ki-67 index of $23 \%$ (high). Postoperatively, prolactin remained elevated $(725-891 \mathrm{ng} / \mathrm{ml}$ ) and cabergoline was increased to $1 \mathrm{mg}$ three times weekly, with serum prolactin further increasing to $3507 \mathrm{ng} / \mathrm{ml}$ five months postoperatively. Repeat MRI revealed extension of the tumor with optic chiasm compression and left orbit invasion. Because of acute left vision loss with ophthalmoplegia, an urgent left frontotemporal craniotomy and tumor resection were conducted. The Ki-67 index of the tumor was $\mathbf{2 4 . 8 \%}$, the mitotic figure immunostain phosphohistone-H3 positive. Sixty percent $(60 \%)$ of tumor cells were positive for p53. Cabergoline was increased to $1 \mathrm{mg}$ daily but prolactin remained elevated $(770 \mathrm{ng} / \mathrm{ml})$. The patient then underwent proton beam radiation to the area of concern involving the sella. Prolactin thereafter improved to $44 \mathrm{ng} / \mathrm{ml}$. He then developed acute vision loss of the right eye with an MRI showing tumor in the right cavernous
\end{abstract}


sinus. A $15 \mathrm{~mm}$ dural-based right temporal mass believed to be a metastasis was also noted. Following this scan, he was considered too high risk for debulking surgery and instead underwent gamma knife irradiation to the sella area. This shrank the right cavernous sinus tumor mass, while the right temporal mass increased in size. The patient developed blindness and left-sided weakness and required enteral feeding and tracheostomy after prolonged intubation. A trial of chemotherapy with temozolomide $(350 \mathrm{mg}$ daily for 5 days) near the end of his life was unsuccessful. He died on home hospice 31 months after his first surgery. CONCLUSION: Headaches, vision changes, and symptoms of androgen deficiency syndrome can be manifestations of an aggressive prolactinoma that might require surgery and additional medical therapy including cabergoline and temozolomide with an unpredictable time of survival.

Key words: Cabergoline, Pituitary carcinoma, Prolactin, Temozolomide

\section{INTRODUCTION}

Malignant prolactinoma is exceedingly rare and cannot be diagnosed on histological grounds alone. Similar to other neuroendocrine tumors such as pheochromocytoma, the mitoses index, Ki-67, p53, and others are utilized in helping to determine whether a tumor is benign or malignant or to better predict tumor behavior. However, for pituitary tumors the presence of metastases within or outside the CNS (distant from the pituitary) is required to characterize a tumor as malignant or to ascertain that the tumor is not contiguous with the primary sellar tumor, ${ }^{1,2}$ while the term "aggressive" should not be used synonymously with "invasive". ${ }^{3}$ Pituitary carcinoma is very uncommon, accounting for approx. $0.1 \%$ of all pituitary tumors. Treatment options are limited. Sur- vival is usually poor. We herein present the unusual case of an unfortunate young man with an aggressive prolactinoma, the complications of which led to his premature death.

\section{CASE REPORT}

A 25-year-old white man presented to his primary care physician with severe headaches, low energy, and decreased libido, without clinical features of Cushing's syndrome. A brain magnetic resonance imaging (MRI) showed a $4 \times 3 \times 2 \mathrm{~cm}$ pituitary tumor invading the left cavernous sinus (Figure 1). Laboratory findings revealed elevated prolactin (diluted $470 \mathrm{ng} / \mathrm{mL}$; normal less than $25 \mathrm{ng} / \mathrm{ml})$, ACTH (82 $\mathrm{pg} / \mathrm{ml}$, normal, 10-60), serum cortisol of $19 \mathrm{mcg} / \mathrm{dl}$, and decreased total testosterone $(176 \mathrm{ng} / \mathrm{dl})$ with
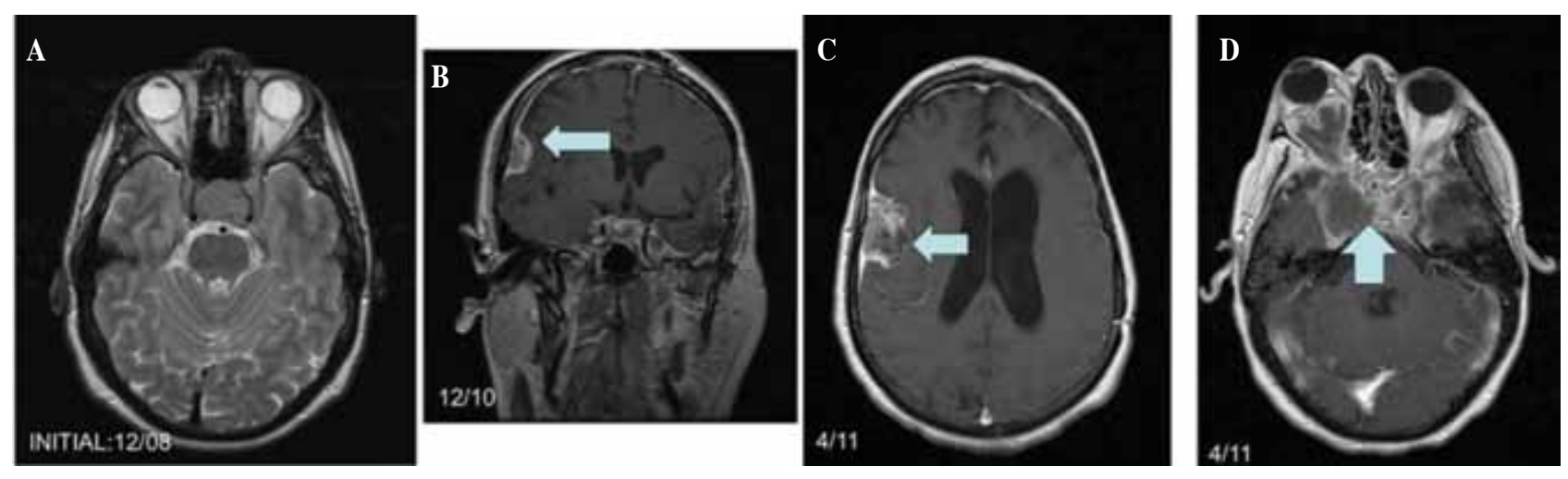

Figure 1. Serial magnetic resonance imaging of the pituitary / brain.
A. Initial MRI, horizontal cut, note sellar area
B. 2-year follow-up, vertical cut, note dural lesion right temporal
C. 28 months follow-up, emphasizing right temporal area
D. 28 months follow-up, emphasizing sellar area. 
low normal LH and FSH. TSH, free T4, and serum cortisol values were normal. Visual fields showed superior quadrantanopia in the left eye. Transsphenoidal pituitary resection was undertaken, taking into consideration the visual field defect and the potential for plurihormonal tumor given the elevated ACTH and prolactin. Cabergoline or bromocriptine were not administered preoperatively. Pathology revealed an atypical pituitary adenoma with increased pleomorphism but no necrosis or mitotic activity with stains diffusely positive for prolactin consistent with prolactinoma (Figure 2). Note was made of atypical cells including multinucleated giant cells and cells with bizarre, enlarged nuclei. The Ki-67 index was $23 \%$ (high) and there was diffuse p53 nuclear labeling. Postoperatively, the patient was discharged on DDAVP (vasopressin), for treatment of diabetes insipidus, and on hydrocortisone, as his morning serum cortisol level was low at $3.6 \mathrm{mcg} / \mathrm{dl}$ with an ACTH of $6 \mathrm{pg} /$ $\mathrm{ml}$. Prolactin remained elevated $(725-891 \mathrm{ng} / \mathrm{ml})$ and cabergoline was increased to $1 \mathrm{mg}$ three times weekly (Table 1). In spite of that, prolactin was $3507 \mathrm{ng} / \mathrm{ml}$ five months postoperatively. Repeat MRI revealed extension of the tumor with optic chiasm compression and left orbit invasion. Surgery was planned, but prior to this he developed acute left vision loss with ophthalmoplegia and underwent urgent left frontotemporal craniotomy and tumor resection at another institution. The Ki-67 labeling index of the tumor was $24.8 \%$. The mitotic figure immunostain phosphohistone-H3 was positive and $60 \%$ of tumor cells were positive for $\mathrm{p} 53$. These outside pathology report parameters compared with the ones from the first resection further document the progression of this pituitary tumor from atypical adenoma to outright carcinoma. The patient's postoperative course was complicated by expressive dysphagia and left CN III, IV, VI nerve palsies, and ischemic brain pathology was attributed to possible intraoperative cerebral vasospasm. Cabergoline was increased to $1 \mathrm{mg}$ daily
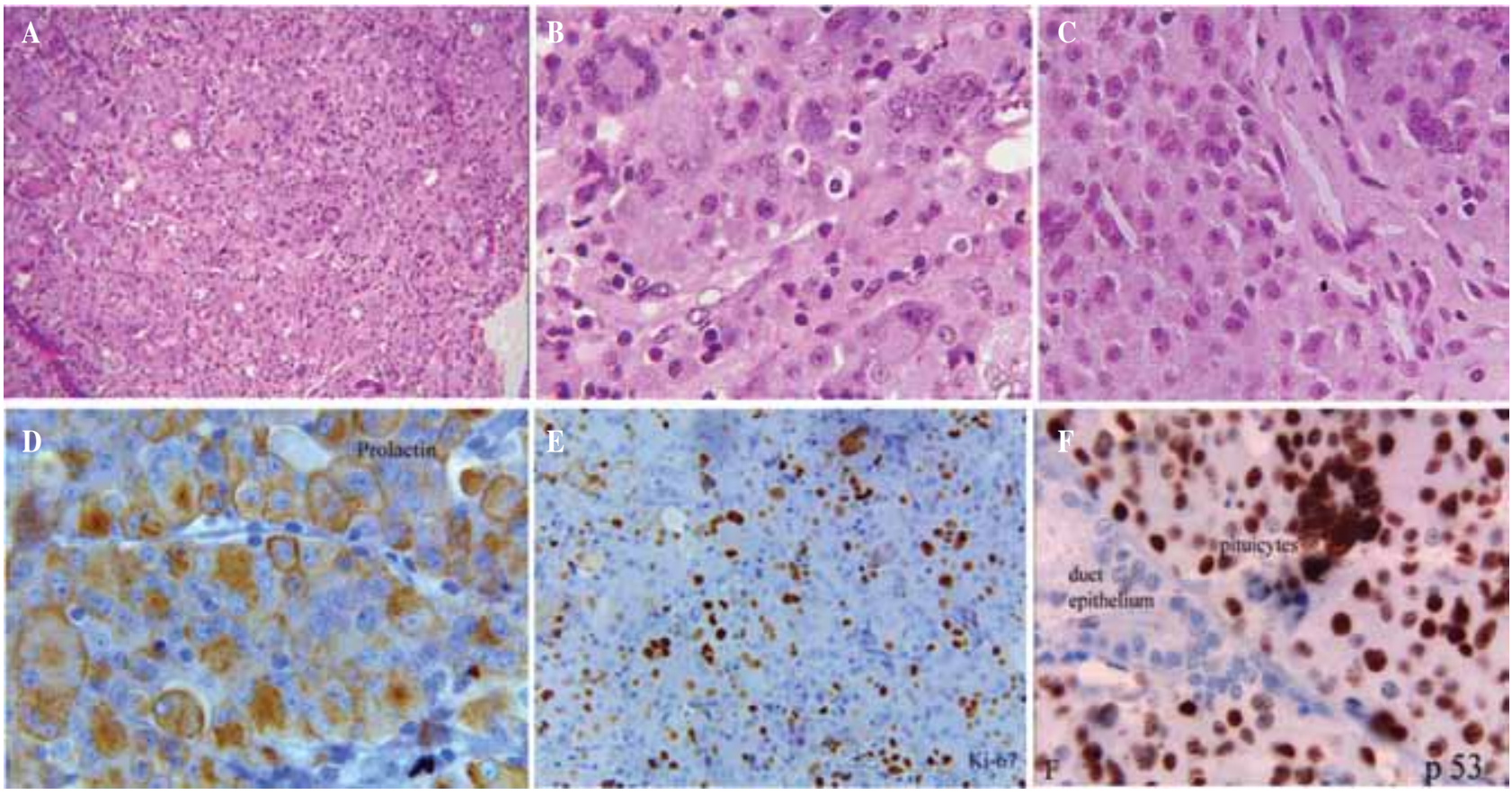

Figure 2. Histology (Hematoxylin \& Eosin) and Immunohistochemistry (prolactin, Ki-67, p53) of atypical pituitary adenoma. A. Acinar or organoid architecture replaced by sheets of cells (low power magnification)

B. Population of pleomorphic nuclei in this area of tumor, including multinucleated giant cells; the tumor cell nuclei have clumped chromatin, huge nucleoli; there is no necrosis (high power magnification)

C. Sheets of cells with pleomorphic nuclei, mitoses are difficult to identify (medium power)

D. Tumor cells are diffusely positive for prolactin

E. High labeling index; $23 \%$ of nuclei are positive for Ki-67

F. Diffuse nuclear labeling with p53 
Table 1. Serial prolactin levels

\begin{tabular}{clc}
\hline Date & Event & $\begin{array}{c}\text { Prolactin } \\
(\mathbf{n g} / \mathbf{m l})\end{array}$ \\
\hline $12 / 29 / 08$ & & 470 \\
$1 / 30 / 09$ & & 725 \\
$3 / 4 / 09$ & & 891 \\
$4 / 6 / 09$ & & 858 \\
$4 / 20 / 09$ & & 1276 \\
$5 / 11 / 09$ & & 470 \\
$6 / 17 / 09$ & & 3507 \\
$6 / 22 / 09$ & & 3593 \\
$8 / 20 / 09$ & $8 / 30 / 09$ craniotomy & 4733 \\
$9 / 21 / 09$ & $12 / 30 / 09$ completion of proton beam & 359 \\
$4 / 19 / 10$ & & 44 \\
$12 / 13 / 10$ & $12 / 2010$ gamma knife irradiation & 835 \\
$3 / 22 / 2011$ & $3 / 22 / 11$ trial of temozolamide & 5552 \\
$7 / 17 / 2011$ & & 3452 \\
\hline
\end{tabular}

on discharge, and levothyroxine and the antiepileptic drug Levetiracetam (Keppra) were added to his regimen. Prolactin remained elevated $(770 \mathrm{ng} / \mathrm{ml})$. He then underwent proton beam radiation (54 Gy in 30 fractions of 1.8 Gy per fraction) to the area of concern involving the sella. Prolactin thereafter improved to $44 \mathrm{ng} / \mathrm{ml}$. He subsequently developed acute vision loss of the right eye (believed to be related to ischemia/ vasospasm) with an MRI showing tumor in the right cavernous sinus (October 2010). A $15 \mathrm{~mm}$ dural-based temporal mass believed to be a metastasis was also noted. Following this scan, he was considered too high risk for debulking surgery and instead underwent gamma knife irradiation while on dexamethasone therapy. This shrank the right cavernous sinus tumor mass, while the right temporal mass increased in size (Figure 1, Dec 2010). The patient developed blindness and left-sided weakness and required enteral feeding and tracheostomy after a prolonged intubation. A trial of chemotherapy with temozolomide $(350 \mathrm{mg}$ daily for 5 days as an inpatient) near the end of his life was unsuccessful. He died on home hospice 31 months after his first surgery.

\section{DISCUSSION}

Similarly to other neuroendocrine tumors (NET) such as pheochromocytoma, the diagnosis of pituitary carcinoma is made only after discovery of metastases or the presence of tumor that is not contiguous with the primary sellar tumor. ${ }^{1-4}$ Proliferation markers Ki-67 labeling index (MIB-1), a monoclonal antibody against the Ki-67 antigen, are used to predict tumor aggressiveness and recurrence. A Ki-67 labeling index cutoff of $3 \%$ has been suggested to predict invasiveness, with higher values being more invasive. ${ }^{3,5} \mathrm{Ki}-67$ is more predictive in functioning tumors than in nonfunctioning tumor. ${ }^{4-6}$ Pituitary carcinomas average a higher Ki-67 index (11\%), although there are reports of carcinomas with a low Ki-67 index, suggesting that there are factors other than proliferation that contribute to aggressiveness and malignant potential. The presence of mitoses and nuclear pleomorphism in a pituitary tumor, like the one presented here in our patient, should raise suspicion for aggressive behavior, although studies differ as to whether these correlate with the proliferation index., ${ }^{3,6}$ The World Health Organization classification of endocrine tumors, including pituitary, requires specific morphologic features and immunophenotypic characteristics (Ki-67 index, p53 staining), as those of our patient, in order to characterize a pituitary tumor as atypical. $^{5}$ Of note, the tumor in our patient already had p53 positivity and histologic features considered to be atypical at first presentation and not only after radiation therapy. Therefore, it appears that detailed histological subtyping represents an excellent predictor of aggressive behavior in addition to analysis of such pituitary tumors for fibroblast growth factor receptor 4, matrix metalloproteinases, pituitary tumor transforming gene, Ki-67, p53, and deletions in chromosome $11 .^{3}$

The molecular pathogenesis of pituitary tumors is still largely unknown despite the fact that several new genes have been identified in familial pituitary tumor syndromes. ${ }^{7-11}$ Consideration of potential hereditary causes or somatic GNAS mutation in pituitary tumorigenesis is important for initial surgical planning and aftercare, including risk for recurrent disease.${ }^{12}$ Recent studies have focused on identifying genetic differences between invasive and noninvasive tumors. In a study using microarray analysis of nonfunctioning pituitary adenomas, overexpression of 4 genes was confirmed in invasive tumors (IGFBP5, MYO5A, FLT3, and NFE2L1) and, given the known effect of the product 
of MYO5A on tumor cell migration and invasion, this may be an area of interest for future research into tumor markers. ${ }^{7}$ Loss of heterozygosity through allelic deletion at 11q13 (the site for the tumor-suppressor gene MEN1), 13q12-14, and at 10q and 1p has been implicated in some pituitary carcinomas. However, as the number of pituitary tumors with described mutations or deletions is small, it is difficult to infer this as a generalized mechanism for aggressive tumor behavior. ${ }^{13} \mathrm{An}$ important point to consider, as in our patient, is the potential of radiation to induce further chromosomal damage to an existing tumor but also normal cells, including pituicytes. Prolactinomas make up approx. 50\% of all pituitary tumors and respond to dopamine agonist therapy, with reduction in size in up to $90 \%$ of (compliant) patients..$^{14}$ For prolactinomas that are resistant to conventional doses of dopamine agonists, high dose cabergoline (12 mg per week or more), surgery, and radiotherapy should be utilized or, if these fail, as in our patient, additional therapies should be used including temozolomide. ${ }^{15,16}$ Pituitary carcinomas and NET can change their hormone expression profile, which in part depends on their grade of differentiation. ${ }^{2,17}$ For instance, von Hippel Lindau disease-associated pheochromocytomas are considered less differentiated than pheochromocytomas in patients with multiple endocrine neoplasia type 2 and have a more norepinephrine/normetanephrine prone biochemical profile. ${ }^{18}$

Resistance to cabergoline (dopamine D2-like receptor agonist) therapy, defined by the lack of normalization of prolactin or failure of the tumor to shrink by $50 \%$, occurs in less than $10 \%$ of patients with prolactinoma. ${ }^{14}$ Histologically, resistant prolactinomas have increased angiogenesis, cellular atypia, proliferation, and invasiveness. There is a spectrum of resistance and most resistant prolactinomas are not carcinomas. Mechanisms for resistance include decreased D2 receptor gene transcription, decreased activity of receptors which regulate D2 receptor expression, and decreased expression of the inhibitory G protein which couples the D2 receptor to adenylyl cyclase. ${ }^{14,19}$ Given the current healthcare state of affairs, one also has to take into account noncompliance with medications that patients are unable to afford before defining the respective tumor as medically refractory. Often, patients feel ashamed to admit to not having taken their drugs because of financial concerns.

Treatment for (truly) dopamine agonist resistant prolactinomas should include transsphenoidal surgery and then radiation for persistent or recurrent disease, although radiotherapy has limited success in these tumors. Advanced medical therapy should be considered for those in whom these efforts fail. ${ }^{19}$ Temozolamide is an oral alkylating agent most often used to treat glioblastoma multiforme which has been shown to be of benefit in some cases of resistant pituitary tumors, including prolactinomas. $(15,16,20,21)$ It interferes with DNA replication via methylation of DNA guanine at the O-6 position. Low levels of the DNA repair enzyme methylguanine methyltransferase (MGMT) may predict a more favorable response to temozolomide (TMZ), ${ }^{22-24}$ although a recent case series suggests otherwise and recommends against using this to guide patient care. In this series and review of the literature, 5 of 6 prolactinomas responded to TMZ, and 3 of 6 had low MGMT staining (2 of 6 with unknown MGMT status). ${ }^{20}$

"Evidence" for rare endocrine tumors is limited and composed of individual case studies. This case highlights the morbidity and mortality associated with aggressive pituitary tumors. Although survival is difficult to predict due to the small number of reported cases, one review estimated survival at eight years from the time of diagnosis and noted only $60 \%$ survival at one year after diagnosis of metastasis. ${ }^{25}$

Further elucidating the pathogenesis of aggressive pituitary tumors, including prolactinomas, will help guide medical treatment options. Earlier diagnosis is needed to commence aggressive treatment prior to the occurrence of metastases. Although in our patient temozolamide was ineffective, it should be considered in patients with aggressive pituitary tumors refractory to standard therapies.

\section{REFERENCES}

1. Heaney AP, 2011 Pituitary carcinoma: difficult diagnosis and treatment. J Clin Endocrinol Metab 96: 3649-3660.

2. Dudziak K, Honegger J, Bornemann A, et al, 2011 Pituitary carcinoma with malignant growth from first presentation and fulminant clinical course - case report and review of the literature. J Clin Endocrinol Metab 96: 2665-2669. 
3. Mete O, Ezzat S, Asa SL, 2012 Biomarkers of aggressive pituitary adenomas. J Mol Endocrinol 49: R69-78.

4. Singer J, Koch CA, Kassahun W, et al, 2011 A patient with a large recurrent pheochromocytoma demonstrating the pitfalls of diagnosis. Nat Rev Endocrinol 7: 749-755.

5. DeLellis RA, Lloyd RV, Heitz PU, Eng C (eds), 2004 World Health Organization Classification of Tumours. Pathology and Genetics of Tumours of Endocrine Organs. Lyon: IARC Press.

6. Turner HE, Wass JA, 1999 Are markers of proliferation valuable in the histological assessment of pituitary tumours? Pituitary 1: 147-151.

7. Galland F, Lacroix L, Saulnier P, et al, 2010 Differential gene expression profiles of invasive and non-invasive non-functioning pituitary adenomas based on microarray analysis. Endocr Relat Cancer 17: 361-371.

8. Boikos SA, Stratakis CA, 2007 Molecular genetics of the cAMP-dependent protein kinase pathway and of sporadic pituitary tumorigenesis. Hum Mol Genet 16: R80-87.

9. Stratakis CA, Tichomirowa MA, Boikos S, et al, 2010 The role of germline AIP, MEN1, PRKAR1A, CDKN1B and CDKN2C mutations in causing pituitary adenomas in a large cohort of children, adolescents, and patients with genetic syndromes. Clin Genet 78: 457-463.

10. Ahmad S, Aaltonen LA, Georgitsi M, et al, 2007 Do single nucleotide polymorphisms in the AIP gene and MEN 1 gene predispose individuals to the development of familial isolated pituitary tumors ? Exp Clin Endocrinol 115: S35-36.

11. Glasker S, Vortmeyer AO, Lafferty AR, et al, $2011 \mathrm{He}-$ reditary pituitary hyperplasia with infantile gigantism. J Clin Endocrinol Metab 96: E2078-2087.

12. Vortmeyer AO, Glasker S, Mehta GU, et al, 2012 Somatic GNAS mutation causes widespread and diffuse pituitary disease in acromegalic patients with McCune-Albright syndrome. J Clin Endocrinol Metab 97: 2404-2413.

13. Gurlek A, Karavitaki N, Ansorge O, Wass JA, 2007 What are the markers of aggressiveness in prolactinomas? Changes in cell biology, extracellular matrix components, angiogenesis, and genetics. Eur J Endocrinol 156: 143-153.

14. Molitch ME, 2008 The cabergoline-resistant prolactinoma patient: new challenges. J Clin Endocrinol Metab 93: 4643-4645.

15. Byrne S, Karapetis C, Vrodos N, 2009 Novel use of temozolomide in a patient with malignant prolactinoma. J Clin Neurosci 16: 1694-1696.

16. Neff L, Weil M, Hedges TR, et al, 2007 Temozolomide in the treatment of an invasive prolactinoma resistant to dopamine agonists. Pituitary 10: 81-86.

17. Miehle K, Tannapfel A, Lamesch P, et al, 2004 Pancreatic neuroendocrine tumor with ectopic adrenocorticotropin production upon second recurrence. J Clin Endocrinol Metab 89: 3731-3736.

18. Koch CA, Mauro D, Walther MM, et al, 2002 Pheochromocytoma in von hippel-lindau disease: distinct histopathologic phenotype compared to pheochromocytoma in multiple endocrine neoplasia type 2. Endocr Pathol 13: 17-27.

19. Oh MC, Aghi MK, 2011 Dopamine agonist-resistant prolactinomas. J Neurosurg 114: 1369-1379.

20. Dillard TH, Gultekin SH, Delashaw JB Jr, Yedinak CG, Neuwelt EA, Fleseriu M, 2011 Temozolomide for corticotroph pituitary adenomas refractory to standard therapy. Pituitary 14: 80-91.

21. Kovacs K, Horvath E, Syro LV, et al, 2007 Temozolomide therapy in a man with an aggressive prolactin-secreting pituitary neoplasm: Morphological findings. Hum Pathol 38:185-189.

22. Bush ZM, Longtine JA, Cunningham T, et al, 2010 Temozolomide treatment for aggressive pituitary tumors: correlation of clinical outcome with $\mathrm{O}(6)$-methylguanine methyltransferase (MGMT) promoter methylation and expression. J Clin Endocrinol Metab 95: E280-290.

23. Raverot G, Sturm N, de Fraipont F, et al, 2010 Temozolomide treatment in aggressive pituitary tumors and pituitary carcinomas: a French multicenter experience. J Clin Endocrinol Metab 95: 4592-4599.

24. Syro LV, Ortiz LD, Scheithauer BW, et al, 2011 Treatment of pituitary neoplasms with temozolomide: a review. Cancer 117: 454-462.

25. Kars M, Roelfsema F, Romijn JA, Pereira AM, 2006 Malignant prolactinoma: case report and review of the literature. Eur J Endocrinol 155: 523-534. 